\title{
Diagnostic Value of Combined Detection of Galectin-3 and N-Terminal B-Type Natriuretic Peptide in Patients with Acute Heart Failure
}

\author{
Huaming Zhang, Jinjun Li, Hongyan Han \\ School of Medicine, Wuhan University of Science and Technology, Wuhan, China \\ Email:maltcose@sina.com
}

How to cite this paper: Zhang, H.M., Li, J.J. and Han, H.Y. (2018) Diagnostic Value of Combined Detection of Galectin-3 and N-Terminal B-Type Natriuretic Peptide in Patients with Acute Heart Failure. World Journal of Cardiovascular Diseases, 8, 208-216.

https://doi.org/10.4236/wjcd.2018.83020

Received: January 27, 2018

Accepted: March 24, 2018

Published: March 27, 2018

Copyright $\odot 2018$ by authors and Scientific Research Publishing Inc. This work is licensed under the Creative Commons Attribution International License (CC BY 4.0).

http://creativecommons.org/licenses/by/4.0/

cc) (i) Open Access

\begin{abstract}
Background: Acute heart failure timely and effective diagnosis and treatment directly affects the prognosis of patients, so early diagnosis of acute heart failure treatment is very important. The current diagnosis of acute heart failure has yet to be further improved. To investigate the relationship between plasma levels of Galectin-3 and NT-proBNP in cardiac structure and function in patients with acute heart failure (AHF) Early detection of failure. Methods: The clinical data of 86 patients with acute heart failure in our hospital were analyzed and followed up. Twenty-six healthy subjects with normal cardiac function were used as control group. The plasma Galectin-3 and NT-proBNP levels were compared between the two groups to observe the value of plasma Galectin-3 combined with NT-proBNP in the diagnosis of acute heart failure. Results: There was no significant difference in the level of Galectin-3 and NT-proBNP between heart function group II and control group, and the levels of cardiac function III and IVG plasma Galectin-3 and NT-proBNP were significantly higher in patients with heart failure Compared with the healthy control group, the patients' LVEF decreased and their cardiac function increased. The levels of plasma Galectin-3 and NT-proBNP increased significantly $(\mathrm{P}<0.01)$. Multivariate Logistic regression analysis demonstrated that plasma levels of Galectin-3 and NT-proBNP were independent of cardiac function. The area under the ROC curve for the combined detection of plasma Galectin-3 and NT-proBNP was greater than the area under the two alone tests. Conclusion: The combined detection of Galectin-3 and NT-proBNP has high sensitivity and specificity in the diagnosis of acute heart failure and can be used as a new detection mode.
\end{abstract}

\section{Keywords}

Galectin-3, N-Terminal B-Type Natriuretic Peptide, Acute Heart Failure 


\section{Introduction}

Acute heart failure is a common clinical critically ill; its timely and effective diagnosis and treatment directly affects the prognosis of patients [1], so early diagnosis of acute heart failure treatment is very important. This study was to follow up the clinical data of 86 patients with acute heart failure and normal healthy people who were treated in our hospital. The purpose of this study was to analyze the clinical significance of combined detection of plasma Galectin-3 and N-terminal B-type natriuretic peptide in the diagnosis of acute heart failure value.

\section{Materials and Methods}

\subsection{Research Objects and Groups}

Eighty-six patients with acute heart failure who were admitted to God-of-Hospitals affiliated to Wuhan University of Science and Technology from January 2014 to December 2016 were included in the criteria: 1) in line with the standard of acute heart failure diagnosed by the cardiovascular branch of the Chinese Medical Association in 2014 [2]; 2) Forrester grading standards [3] clinical grading levels II-IV; 3) patients and their families informed consent and signed informed consent; 4) in line with medical ethics requirements. Exclusion criteria: 1) patients with unstable vital signs; 2) patients with respiratory failure; 3) patients with severe liver and kidney dysfunction; 4) patients with severe infection; 5) patients with mental disorders. Among the patients enrolled in the study, 46 were male and 40 were female, with a mean age of $65 \pm 4.5$ years between the ages of 50 and 80 years. Among them, 40 cases of ischemic cardiomyopathy, 18 cases of hypertensive heart disease, 10 cases of heart valve disease, 8 cases of dilated cardiomyopathy, 6 cases of rheumatic heart disease, 4 cases of hypertrophic cardiomyopathy, heart Features are graded according to Forrester's grading standards. In the meantime, 86 healthy subjects with normal cardiac function were selected as the control group, including 46 males and 40 females, aged from 40 to 58 years with an average age of $50 \pm 6.6$ years. There was no significant difference in general data between the two groups $(\mathrm{P}>0.05)$, which was comparable.

Acute heart failure patients with chronic complications, with chronic renal insufficiency in 7 patients, accounting for $8.1 \%, 9$ cases of patients with hyperlipidemia, accounting for $110.5 \%, 17$ cases of diabetes mellitus, accounting for $19.8 \%$. Cardiac myopathy (37.1\%), coronary heart disease (35.2\%), and valvular heart disease (17.8\%) were the major predictors of new-onset acute heart failure. A total of 18 patients with acute myocardial infarction, $45.0 \%$ of patients with coronary heart disease. Infection was the predominant cause of heart failure (44\%) in the triggers of acute heart failure, followed by myocardial ischemia (27.8\%), excessive physical exertion and emotional agitation (17.9\%), acute arrhythmia (14.9\%), (7.5\%), severe anemia (3.2\%), hypersecretion (7.9\%), hypofunction $(9.9 \%)$, acid-base balance and electrolytes disturbance of pulmonary embolism (2.0\% Hypertensive emergency (1.0\%). All patients were included in 
the clinical manifestations of cardiac insufficiency, left heart failure performance, dyspnea, chest tightness, suffocation more than $90 \%$, about $2 / 3$ of patients with fatigue, cough, sputum. Right heart failure performance than left ventricular failure performance, lower extremity edema $59.2 \%, 53 \%$ of ascites. Physical examination, the vital signs, the general population average respiratory rate $19 \pm 3.8$ beats $/ \mathrm{min}$, the average heart rate $95 \pm 25.2$ beats $/ \mathrm{min}$, the average systolic blood pressure $119.2 \pm 25.9 \mathrm{mmHg}$, mean arterial diastolic blood pressure $75.6 \pm 16.3 \mathrm{mmHg}$. Nearly $40 \%$ of patients had arrhythmia, $20.9 \%$ of patients had heart murmur, $41.6 \%$ of patients had pulmonary sound, of which wet tone (33.7\%) based. Remaining circulation of jugular venous filling and hepatic jugular vein reflux less, less than $10 \%$. Healthy control group without special discomfort and abnormal signs.

\subsection{Method}

Fasting blood samples were collected on the next day $5 \mathrm{ml}$ elbow venous blood, placed in EDTA anticoagulant tubes were stored within $2 \mathrm{~h}$ after the collection, $4^{\circ} \mathrm{C}, 3000 \mathrm{r} / \mathrm{min}$ centrifugation $20 \mathrm{~min},-80^{\circ} \mathrm{C}$ frozen to avoid repeated freezing and thawing. Application BG medicine's Galectin-3 ELISA test kit according to kit instructions for testing. Using electrochemiluminescence immunoassay, the patient's plasma NT-proBNP was detected with a kit from Roche Diagnostics of Germany. Application of Philips EPIQ7 color ultrasound system for cardiac color Doppler ultrasound to assess the patient's systolic function.

\subsection{Statistical Methods}

SPSS 22.0 software was used for statistical analysis. The $t$ test was used to measure the data between the two groups. Chi-square test was used to count data. Logistic regression was used to analyze the risk factors for screening acute heart failure. The test level was 0.05 . When $\mathrm{P}<0.05$, has statistical significane. The working curve (ROC curve) of subjects tested separately and jointly with Galectin-3 and NT-proBNP was established to calculate their specificity and sensitivity.

\section{Results}

\subsection{Heart Failure Group and Control Group Left Ventricular Ejection Fraction (LVEF) and Plasma Levels of Galectin-3 and NT-proBNP Compared}

Heart failure group LVEF increased significantly compared with the control group, the difference was statistically significant $(\mathrm{P}<0.01$, Table 1$)$. Plasma levels of Galectin-3 and NT-proBNP in HF group were significantly higher than those in control group $(\mathrm{P}<0.01$, Table 1$)$

\subsection{Different Cardiac Function LVEF, Plasma Galectin-3 and NT-proBNP Levels Compared}

There was no significant difference in levels of Galectin-3 and NT-proBNP between NYHA Class II and control groups. Plasma levels of Galectin-3 and 
Table 1. Heart failure group and control group heart function and plasma levels of Galectin-3 and NT-proBNP.

\begin{tabular}{ccccc}
\hline Groups & Cases & NT-proBNP(ng/L) & Galectin-3(ug/L) & LVEF(\%) \\
\hline Experimental group & 86 & $2044.86 \pm 379.01$ & $19.42 \pm 4.76$ & $38.14 \pm 10.76$ \\
Control group & 86 & $251.45 \pm 95.47$ & $10.27 \pm 1.89$ & $59.51 \pm 9.98$ \\
t value & & 40.47 & 21.63 & 5.34 \\
P value & & 0.006 & 0.004 & 0.007
\end{tabular}

NT-proBNP in patients with NYHA Class III-IV were significantly higher than those in the control group, the difference was statistically significant. The levels of Galectin-3 and NT-proBNP in patients with heart failure were significantly lower with the increase of cardiac function grade, and the difference was statistically significant $(\mathrm{P}<0.01$, Table 2$)$.

\subsection{Different Cardiac Function in Plasma Levels of Galectin-3 and NT-proBNP Multivariate Logistic Regression Analysis}

Multivariate Logistic regression analysis showed that LVEF, plasma Galectin-3 and NT-proBNP were the dependent variables of heart function (no heart failure $=0$, heart failure $=1$ ). The analysis showed that the high left ventricular ejection fraction Plasma levels of Galectin-3 and NT-proBNP were risk factors for cardiac function $(\mathrm{OR}=2.963,2.374,1.987,95 \% \mathrm{CI}$ : $1.374-3.836,2.105-5.974$, 1.402 - 3.519, respectively, $\mathrm{P}<0.01$ ). See Table 3.

\subsection{Galectin-3 and NT-proBNP Single Test and the Accuracy of the Joint Test}

Using Galectin-3 and NT-proBNP as the test variables, whether the occurrence of heart failure as a state variable, the definition of the state variable is 1 , the establishment of the working curve of patients (ROC), curve results show that the separate detection of Galectin-3 and NT-proBNP in the diagnosis Acute heart failure has good sensitivity and specificity. Galectin-3 is more sensitive than NT-proBNP, but its specificity is not high. When the two were combined, the sensitivity and specificity were significantly improved, the difference was statistically significant (Table 4).

\section{Discussion}

In clinical work, the diagnosis and differential diagnosis of acute heart failure is still a major problem, easily lead to missed diagnosis. Rapid progress of acute heart failure, once the condition deteriorated, often endanger the lives of patients. Acute heart failure with poor prognosis [3], in-hospital mortality was 3\%, $60 \mathrm{~d}$ mortality was $9.6 \%, 3$-year and 5-year mortality rates were $30 \%$ and $60 \%$, respectively, and acute myocardial infarction caused by acute heart failure Fatality higher. In recent years, NT-ProBNP and Galectin-3 have become hot spots in the early diagnosis of heart failure [4] [5] [6] [7] [8]. This study aimed to explore 
Table 2. Different cardiac function LVEF, plasma Galectin-3 and NT-proBNP levels.

\begin{tabular}{ccccc}
\hline Groups & Cases & NT-proBNP(ng/L) & Galectin-3(ug/L) & LVEF(\%) \\
\hline Control group & 86 & $251.45 \pm 95.47$ & $10.27 \pm 1.89$ & $59.51 \pm 9.98$ \\
NYHA Class II & 13 & $394.35 \pm 87.54$ & $10.51 \pm 3.10$ & $42.77 \pm 12.41$ \\
NYHA Class III & 52 & $1747.60 \pm 324.36$ & $13.52 \pm 5.08$ & $38.67 \pm 10.93$ \\
NYHA Class IV & 21 & $3678.86 \pm 357.961$ & $19.62 \pm 3.98$ & $33.81 \pm 10.41$ \\
\hline
\end{tabular}

Note: $\mathrm{P}>0.05$ compared with NYHA Class II, $\mathrm{P}<0.05$ compared with NYHA Class III, $\mathrm{P}<0.05$ compared with NYHA Class IV.

Table 3. Different cardiac function risk factors in multivariate Logistic regression analysis.

\begin{tabular}{ccccc}
\hline \multirow{2}{*}{ Fators } & P value & OR & \multicolumn{2}{c}{$95 \% \mathrm{CI}$} \\
\cline { 4 - 5 } & & & Lower Bound & Upper Bound \\
\hline LVEF & 0.00 & 2.963 & 1.374 & 3.836 \\
NT-proBNP & 0.03 & 2.374 & 2.105 & 2.974 \\
Galectin-3 & 0.01 & 1.987 & 1.402 & 3.519 \\
\hline
\end{tabular}

Table 4. NT-proBNP with Galectin-3 separate test and the accuracy of the joint check.

\begin{tabular}{cccc}
\hline Indicators & NT-proBNP & Galectin-3 & NT-proBNP + Galectin-3 \\
\hline AUC & 0.886 & 0.895 & 0.932 \\
Sensitivity (\%) & 86.4 & 87.3 & 91.3 \\
Specificity (\%) & 88.5 & 70.5 & 90.5 \\
95\%CI & $0.835-0.937$ & $0.841-0.948$ & $0.890-0.974$ \\
\hline
\end{tabular}

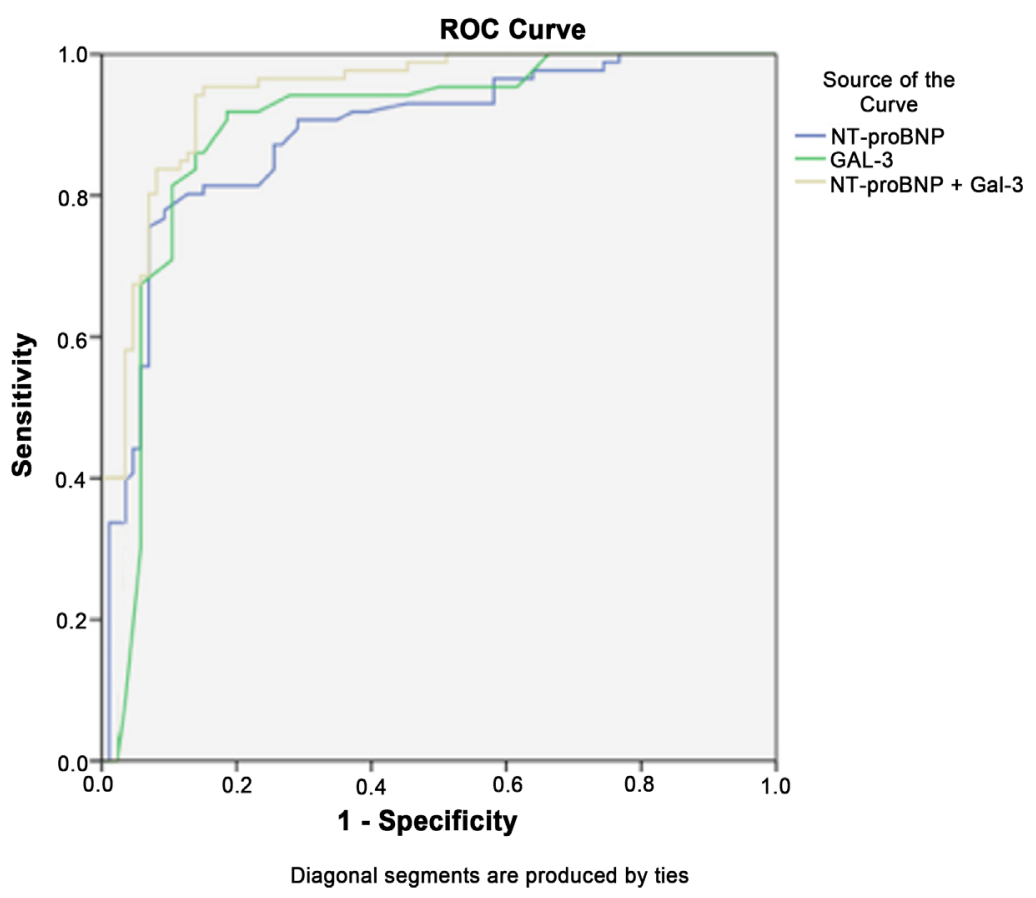


the value of galectin- 3 and $\mathrm{N}$-terminal B-type natriuretic peptide in the diagnosis of acute heart failure.

A large number of studies have shown that BNP can better reflect the function of the heart [1], making it widely used in the diagnosis of heart failure. BNP is a chemical that is pulled in the ventricular wall and released into the blood by cardiomyocytes [9] [10]. BNP is also present in normal tissues and is present in very low plasma concentrations. Cardiomyocytes release BNP while also releasing equimolar NT-ProBNP into the bloodstream. Compared with BNP, NT-ProBNP has higher stability in blood plasma, longer half-life and is less affected by other substances. As the heart expands, the hemodynamics and neuroendocrine activities in the heart cavity change. The higher the pressure in the ventricle, the stronger the ventricular wall is pulled and the higher the level of NT-ProBNP secreted by cardiomyocytes [11]. In the present study, the ejection fraction of patients with heart failure was significantly lower than that of the control group, while the level of NT-proBNP was significantly higher than that of the control group, confirming that NT-proBNP has a very important clinical value in the diagnosis of acute heart failure. Nomura et al. [12] showed that serum creatinine and NT-proBNP were positively correlated. Studies have shown that eGFR is an independent factor of NT-proBNP, eGFR decreased $10 \mathrm{ml} / \mathrm{min}$, NT-proBNP increased 37.7\% [13] [14]. The level of NT-proBNP after stratified by age and renal function can be used as a good observation index.

The study found that [15], inflammation can aggravate myocardial injury, thereby worsening cardiac function, plays a very important role in ventricular remodeling. Most of the inflammatory cytokines have a negative inotropic effect, thereby reducing myocardial contractility and cardiac output, worsening cardiac function [16] [17]. In recent years, studies have shown [18] [19] [20] [21]: Galectin-3 can cause cardiac hypertrophy caused by inflammatory stimuli, resulting in decreased myocardial compliance, and thus cardiac dysfunction. The higher the level of Gal-3, the worse heart function [22]. Galectin-3 can stimulate neutrophil activation and adhesion in the acute inflammatory response and promote inflammation. Sato S et al. [23] detected a large number of Galectin-3 deposits in pneumococcal infected alveoli accompanied by neutrophils A large number of Galectin-3 in alveolar macrophages cultured with Streptococcus pneumoniae membrane isolates; and in chronic inflammatory reaction can stimulate the activation of mononuclear macrophages and fibroblasts and promote the process of fibrosis, Such as chronic pancreatitis, heart failure, pulmonary fibrosis, renal disease-related tissues can detect the expression of GAL-3 [24], In patients with HF complicated with renal insufficiency, elevated levels of galectin-3 in the blood are associated not only with heart release during heart failure but also with renal release during renal insufficiency. Therefore, high levels of Galectin in patients with HF and renal insufficiency -3 may not be able to fully reflect the severity of cardiac function. Gopel et al. found that patients with or without heart failure Galectin-3 were negatively correlated with glomerular filtration rate, elevated Galectin-3 and renal insufficiency are closely related, and this correlation 
is not affected by heart failure Degree of influence [25].

\section{Conclusion}

In this study, our results showed that plasma levels of Galectin-3 and NT-proBNP in patients with heart failure were significantly higher than those in healthy controls, both of which could be used as indicators of acute heart failure. There was no significant difference in the levels of Galectin-3 and NT-proBNP between the heart function class II and the control group, both of which had the risk of misdiagnosis. In clinical practice, the symptoms of patients with heart function class II are often not obvious, thus greatly increasing the risk of missed diagnosis and requiring extra vigilance at work. The levels of plasma Galectin-3 and NT-proBNP in patients with grade III and IV plasma levels were significantly higher than those in healthy controls. The levels of plasma Galectin-3 and NT-proBNP were increased with the decrease of left ventricular ejection fraction. The level was significantly increased $(\mathrm{P}<0.01)$. Multivariate logistic regression analysis demonstrated that plasma Galectin-3 and NT-proBNP were independent of cardiac function. ROC curve results show that the detection of Galectin-3 and NT-proBNP alone in the diagnosis of acute heart failure has a good sensitivity and specificity. Galectin-3 is more sensitive than NT-proBNP, but its specificity is not high. In addition, Galectin-3 is elevated in both heart failure and in vivo, and pulmonary infection is a common predisposing factor in acute left heart failure Galectin-3 is even less specific for the diagnosis of acute heart failure when it is present in the body. NT-ProBNP is even better at acute heart failure with renal insufficiency. Therefore, when the two jointly examined, the sensitivity and specificity are significantly improved, there is an important clinical value.

\section{References}

[1] Brar, S., McAlister, F.A., Youngson, E., Rowe, B.H., et al. (2013) Do Outcomes for Patients with Heart Failure Vary by Emergency Department Volume? Circulation: Heart Failure, 6, 1147-1154. https://doi.org/10.1161/CIRCHEARTFAILURE.113.000415

[2] Cardiovascular Diseases Section of Chinese Medical Association Editorial Board of Chinese Journal of Cardiovascular Diseases Guideline for Diagnosis and Treatment of Heart Failure 2014. Chinese Journal of Cardiovascular Diseases, 42, 98-122.】

[3] Forrester, J.S., Diamond, G.A. and Swan, H.J. (1977) Correlative Classification of Clinical and Hemodynamic Function after Acute Myocardial Infarction. American Journal of Cardiology, 39, 137-145. https://doi.org/10.1016/S0002-9149(77)80182-3

[4] Bao, J., Ding, R., Zou, L., et al. (2016) Forsythiae Fructus Inhibits B16 Melanoma Growth Involving MAPKs/Nrf2/HO-1 Mediated Anti-Oxidation and Anti-Inflammation. The American Journal of Chinese Medicine, 44, 1043-1061. https://doi.org/10.1142/S0192415X16500580

[5] Yancy, C.W., Jessup, M., Bozkurt, B., Butler, J.C., et al. (2013) ACCF/AHA Guideline for the Management of Heart Failure: Executive Summary: A Report of the American College of Cardiology Foundation/American Heart Association Task 
Force on Practice Guidelines. Circulation: An Official Journal of the American Heart Association, 16.

[6] Oremus, M., Don-Wauchope, A., McKelvie, R., et al. (2014) BNP and NT-proBNP as Prognostic Markers in Persons with Chronic Stable Heart Failure. Heart Failure Reviews, 19, 471-505. https://doi.org/10.1007/s10741-014-9439-6

[7] Roberts, E., Ludman, A.J., Dworzynski, K., et al. (2015) The Diagnostic Accuracy of the Natriuretic Peptides in Heart Failure: Systematic Review and Diagnostic Meta-Analysis in the Acute Care Setting. BMJ, 350, h910. https://doi.org/10.1136/bmj.h910

[8] Balion, C., McKelvie, R., Don-Wauchope, A.C., et al. (2014) B-Type Natriuretic Peptide-Guided Therapy: A Systematic Review. Heart Failure Reviews, 19, 553-564. https://doi.org/10.1007/s10741-014-9451-x

[9] Elisabetta, C., Chiara, M., Elena, L., et al. (2015) Endogenous Ouabain: An Old Cardiotonic Steroid as a New Biomarker of Heart Failure and a Predictor of Mortality after Cardiac Surgery. BioMed Research International, 20, 714.

[10] Palazzuoli, A., Gallotta, M., Quatrini, I., et al. (2010) Natriuretic Peptides (BNP and NT-proBNP): Measurement and Relevance in Heart Failure. Vascular Health and Risk Management, 1, 411-418. https://doi.org/10.2147/VHRM.S5789

[11] Sata, Y. and Krum, H. (2010) The Future of Pharmacological Therapy for Heart Failure. CIRC, 74, 809-817.

[12] Nomura, H., Hayashi, T., Esaki, T., et al. (2002) Standardization of Plasma Brain Natriuretic Peptidec Concentrations in Older Japanese-Relationship to Latent Renal Dysfunction and Ischemic Heart Disease. Journal of the American Geriatrics Society, 50, 1504-1509. https://doi.org/10.1046/j.1532-5415.2002.50405.x

[13] Vickery, S., Price, C.P., John, R.I., et al. (2005) B-Type Natriuretic Peptide (BNP) and Amino-Terminal proBNP in Patients with CKD: Relationship to Renal Function and Left Ventricular Hypertrophy. American Journal of Kidney Diseases, 46, 610-620. https://doi.org/10.1053/j.ajkd.2005.06.017

[14] Forfia, P.R., Watkins, S.P., Rame, J.E., et al. (2005) Relationship between Btype Natriuretic Peptides and Pulmonary Capillary Wedge Pressure in the Intensive Care Unit. Journal of the American College of Cardiology, 45, 1667-1671. https://doi.org/10.1016/j.jacc.2005.01.046

[15] Kalogeropoulos, A.P., Georiopoulou, V.V. and Butler, J. (2012) From Risk Factors to Structural Heart Disease: The Role of Inflammation. Heart Failure Clinics, 8, 113-123. https://doi.org/10.1016/j.hfc.2011.08.002

[16] De Boer, R.A., Voors, A.A. and Muntendam, P. (2009) Galectin-3: A Novel Mediator of Heart Failure Development and Progression. European Journal of Heart Failure, 11, 811-817. https://doi.org/10.1093/eurjhf/hfp097

[17] Van Den Borne, S.W., Diez, J. and Blankesteijn, W.M. (2010) Myocardial Remodeling after Infarction: The Role of Myofibroblasts. Nature Reviews Cardiology, 7, 30-37. https://doi.org/10.1038/nrcardio.2009.199

[18] Lok, D.J., et al. (2013) Galectin-3 Is an Independent Marker for Ventricular Remodeling and Mortality in Patients with Chronic Heart Failure. Clinical Research in Cardiology, 102, 103-110.

[19] De Boer, R.A., Edelmann, F. and Cohen Solal, A. (2013) Galectin-3 in Heart Failure with Preserved Ejection Fraction. European Journal of Heart Failure, 15, 1095-1101. https://doi.org/10.1093/eurjhf/hft077

[20] Sato, S., Ouellet, N., Pelletier, I., et al. (2002) Role of Galectin-3 as an Adhesion Mo- 
lecule for Neutrophil Extravasation during Streptococcal Pneumonia. Journal of Immunology, 168, 1813-1822. https://doi.org/10.4049/jimmunol.168.4.1813

[21] Saivagno, G.I., Schena, F., Geiati, M., et al. (2014) The Concentration of High-Sensitivity Troponin 1, Gaiectin-3 and NT-proBNP Substantially Increase after a60-km Ultramarathon. Clinical Chemistry and Laboratory Medicine, 52, 267-272.

[22] Gopal, D.M., Kommineni, M., Ayalon, N., Koelbl, C., Ayalon, R., Biolo, A., Dember, L.M., Downing, J., Siwik, D.A., Liang, C.S., et al. (2012) Relationship of Plasma Galectin-3 to Renal Function in Patients with Heart Failure: Effects of Clinical Status, Pathophysiology of Heart Failure, and Presence or Absence of Heart Failure. Journal of the American Heart Association, 1, e000760.

https://doi.org/10.1161/JAHA.112.000760

[23] Carrasco Sanchez, F.J., Aramburu Bodas, O. and Salamanca Bautista, P. (2013) Predictive Value of Serum Galectin-3 Levels in Patients with Acute Heart Failure with Preserved Ejection Fraction. International Journal of Cardiology, 169, 177-182. https://doi.org/10.1016/j.ijcard.2013.08.081

[24] Shah, R.V., Chen Tournoux, A.A. and Picard, M.H. (2010) Galectin-3, Cardiac Structure and Function, and Long-Term Mortality in Patients with Acutely Decompensated Heart Failure. European Journal of Heart Failure, 12, 826-832. https://doi.org/10.1093/eurjhf/hfq091

[25] Van Der Velde, A.R., Gullestad, L., Ueland, T., et al. (2013) Prognostic Value of Changes in Galectin-3 Levels over Time in Patients with Heart Failure: Data from Corona and Coach. Circulation: Heart Failure, 6, 219-226. https://doi.org/10.1161/CIRCHEARTFAILURE.112.000129 